\title{
II. DIGESTION DES PROTÉINES ET ABSORPTION INTESTINALE DES ACIDES AMINÉS
}

\author{
I N T R O D U C T I O N
}

\author{
A. FRANÇOIS \\ Station centrale de Nutrition, \\ Centre national de Recherches zootechniques, fouy-en-fosas (Seine-et-Oise).
}

Les acides aminés doivent être apportés par les aliments en proportion convenable et équilibrée pour couvrir les besoins de l'organisme. Toutefois, pour qu'ils atteignent leur efficacité maximum, cette condition n'est pas suffisante : les acides aminés doivent en outre arriver simultanément sur les lieux de la synthèse protéique. Il en résulte qu'une digestion incomplète des protéines ou qu'une absorption partielle de certains acides aminés affecterait l'utilisation des protéines; leur efficacité serait diminuée et le résultat pratique serait identique au cas où l'apport alimentaire d'acides aminés est insuffisant ou déséquilibré.

Ce fait souligne la nécessité de mieux connaître la chronologie du passage des différents acides aminés à travers la paroi intestinale, et ceci en fonction de différents facteurs tels que la nature de la protéine ou la composition du reste de la ration. En première approximation, cet aspect cinétique peut être étudié en analysant le sang prélevé dans la veine porte à des temps différents après un repas. L'étude de la variation de la concentration du sang en acides aminés devrait évidemment être complétée par la mesure du débit afin de permettre l'établissement d'un bilan. L'expérience effectuée chez le porc par Pion, FaUconneau et Rerat a montré de très sensibles différences dans le comportement de chacun des acides aminés étudiés. La concentration du sang porte, peut être multiplié par 3 ou 5 dans le cas de certains acides aminés indispensables ou semi-indispensables, alors que pour certains autres, la variation est insensible. Cette voie de recherche semble donc prometteuse, mais, évidemment le fait que des synthèses de protéines peuvent survenir dans la paroi intestinale complique l'interprétation de ces résultats. La vitesse de transit des aliments est, par ailleurs, un facteur qui peut influer sur la digestion des protéines et l'absorption des acides aminés. L'étude de 
Rerat et Lougnon, qui comporte une étude analytique de la composition des contenus digestifs à différents niveaux du tractus et à des temps différents après le repas, apporte une première contribution dans ce domaine.

L'influence de la flore du tube digestif du monogastrique a été mise en évidence depuis plusieurs années au Laboratoire des Métabolismes du C.N.R.Z. La flore intestinale peut en effet désaminer ou décarboxyler les acides aminés et les dégrader ainsi très profondément. Toutefois, ces recherches ne font pas l'objet de la présente action concertée et, en conséquence, ne sont rappelées ici que pour mémoire (cf. Michel M., Ann. Biol. anim, Bioch. Biophys., 1961, 1; 16-28; 213-221).

Les bases théoriques de l'ensemble de ces recherches doivent, sur le plan pratique, permettre une meilleure évaluation des besoins du porc en acides aminés. 